\title{
Prolapsing Bladder Polyp Causing Bladder Outlet Obstruction in a Neonate-
}

\section{A Case Report}

\author{
Aamer Iqbal Ali ${ }^{1}$, Santosh V Patil ${ }^{1}$, Dinesh H Kittur ${ }^{1}$, Sudhakar S Jadhav ${ }^{1}$ and Kailas Bhandarkar ${ }^{* 2}$ \\ ${ }^{1}$ Sushrut Jadhav Kinderchirurgie Charitable Trust's Paediatric Surgery Centre and PG Institute, Sangli, India \\ ${ }^{2}$ Department of Paediatric Surgery, Evelina London Children's hospital, UK
}

"Corresponding author: Bhandarkar K, Department of Paediatric Surgery, Evelina London Children's hospital, London, UK, Tel: +447551974013; E-mail: drbhandarkar@hotmail.com

Received: October 22, 2018; Accepted: October 28, 2018; Published: October 31, 2018

\begin{abstract}
Masses arising from the urinary bladder can cause obstructive uropathy. Rarely these masses can protrude from the urethral meatus. Here we discuss a neonate who presented with a bladder mass prolapsing from his external meatus with signs and symptoms of obstructive uropathy. He was investigated and diagnosed to have bladder mass. He recovered completely after suprapubic excision of mass. Histopathology revealed fibroepithelial polyp of bladder. The clinical presentation, differential diagnosis and management is discussed.
\end{abstract}

Keywords: Fibro-epithelial polyp of the urethra; Bladder mass; Obstructive uropathy; Congenital

\section{Introduction}

Congenital masses arising from the urinary bladder in neonates are exceptional [1]. Urethral polyps are a rare anomaly of the male urethra. Presentation with prolapsing mass from the external urethral meatus is rare [2]. They may present with signs and symptoms of obstructive uropathy, upper tract dilation, and azotemia [3]. Management include observation or excision of via a suprapubic or transurethral techniques [1,3].

\section{Case Report}

A one month old male born at term presented with poor urinary stream and dribbling since birth. Antenatal renal and bladder ultrasound were normal. Mother also gave a history of a mass prolapsing through the external meatus. On examination, the neonate did not have any dysmorphic features. His belly was distended with a palpable bladder. Blood investigations revealed urea of 48 and serum creatinine of $1.3 \mathrm{mg}$ per dl. Renal ultrasound demonstrated bilaterally dilated pelvicalyceal systems, dilated ureters, bilateral perinephric urinomas and a thick walled bladder with a mass. Micturating cystourethrogram showed bilateral grade four vesicoureteric reflux (VUR) with bilateral urinomas and thick walled bladder with a filling defect

Citation: Ali AI, Patil SV, Bhandarkar K, et al. Prolapsing Bladder Polyp Causing Bladder Outlet Obstruction in a Neonate- A Case Report. Clin Case Rep Open Access. 2018;1(2):112.

(C)2018 Yumed Text. 
(FIG. 1). The anterior and posterior urethra was normal. Initial management included draining the bladder with a urethral catheter, careful maintenance intravenous fluids and accurate fluid intake and output monitoring.

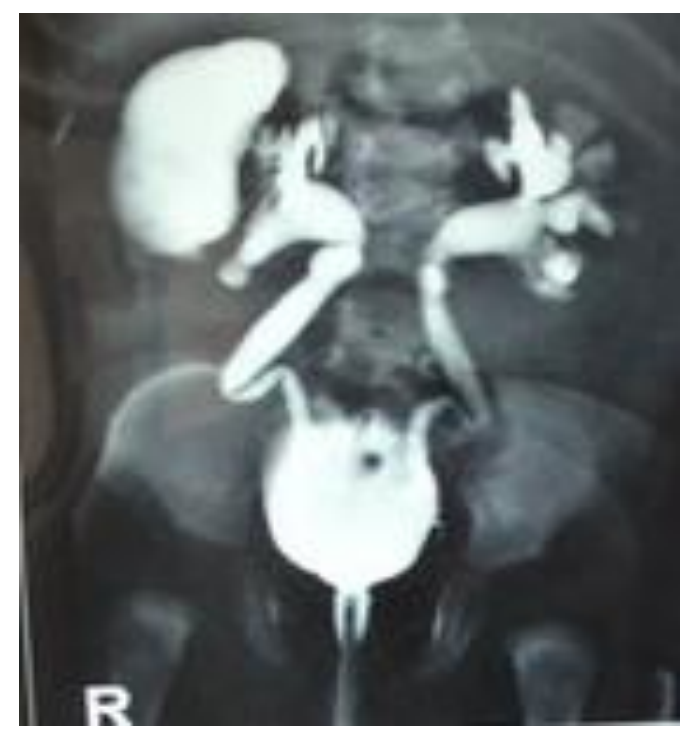

FIG. 1. MCUG showing bilateral vesicoureteric reflux, bilateral perinehric urinomas and a filling defect in the dome of the bladder.

After stabilisation, a cystoscopy was performed. Cystoscopy showed a normal anterior and posterior urethra, a trabeculated bladder and bilateral wide open ureteric orifices and a large pedunculated bladder mass arising from the dome(FIG. 2). Since the mass was large for transurethral excision we decided to go ahead with transvesical excision (FIG. 3 and 4). Bladder was drained via a suprapubic cystotomy. Histopathology revealed a fibroepithelial polyp of bladder. Follow up scans showed complete resolution of bilateral hydronephrosis and urinomas. Follow up cystogram did not show any VUR. Urea and creatinine returned to normal baseline values with a month of the procedure.

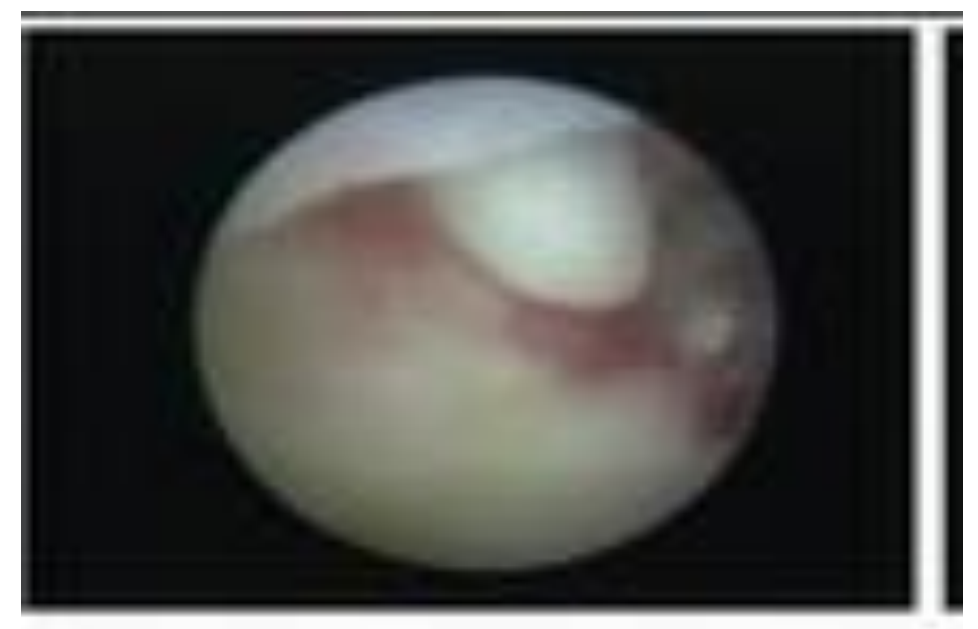

FIG. 2. Cystoscopic view showing a polypoid mass in the bladder. 


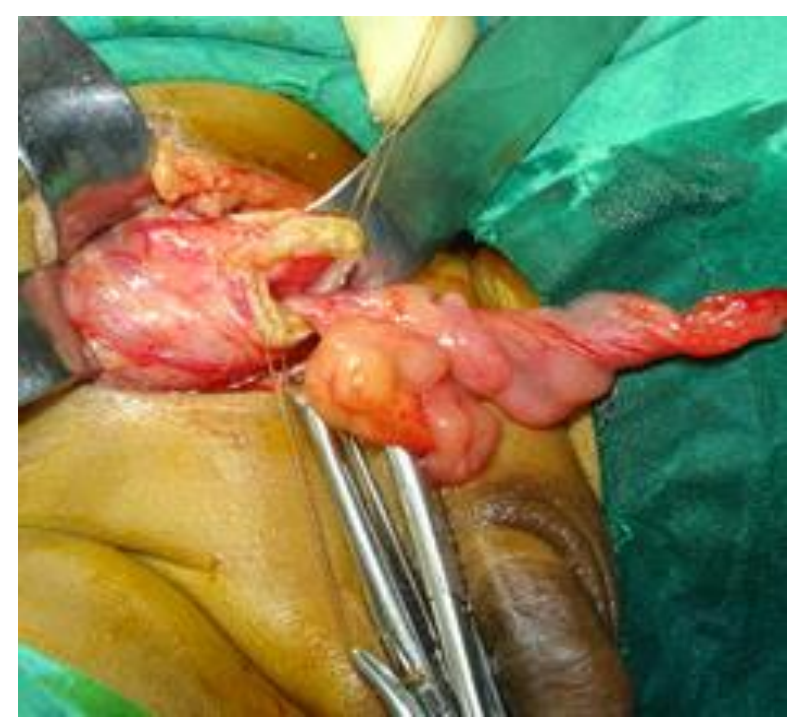

FIG. 3. Suprapubic excision of the bladder polyp.

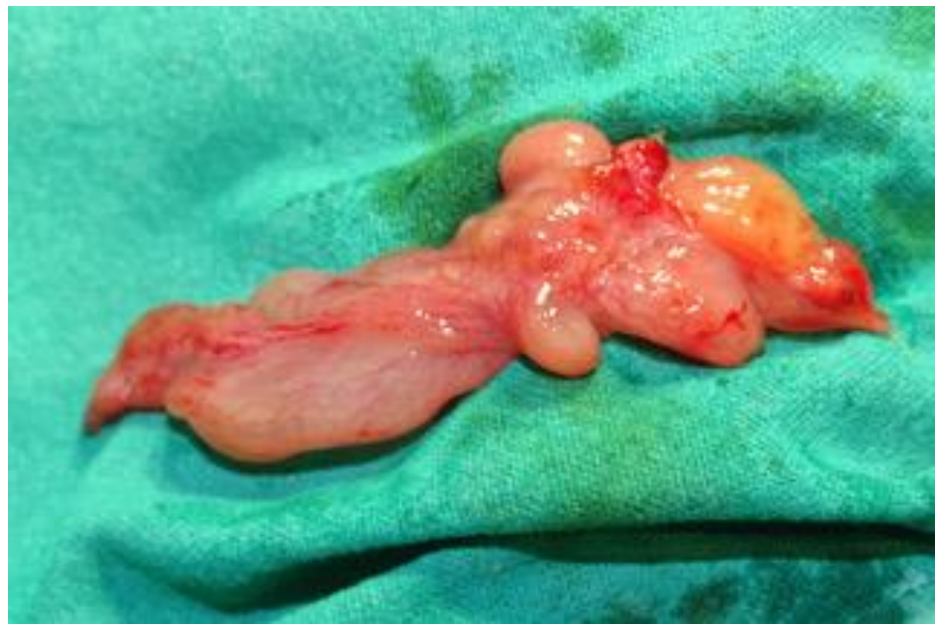

FIG. 4. Resected specimen.

\section{Discussion}

Fibroepithelial polyps of the urethra are rare benign epithelial tumours, more frequently encountered in males. They may prolapse out of the urethra in neonates but this is rare [3]. These masses may result in obstructive uropathy, urinary ascites, vesico-ureteric reflux, renal insufficiency [2] or with features of voiding dysfunction [4]. Although the exact incidence is unknown, fibroepithelial polyps are important differential diagnosis of lower urethral obstruction in male children. Rarely this could be a presenting feature of Beckwith-Wiedemann syndrome [4].

Currently an increased incidence has been reported during the past 2 deacdes, owing to an increased use of voiding cystourethrography [2]. However, presentation in the newborn population is extremely rare. Common differential diagnosis in this age group include both benign and malignant masses. They include prolapsed ectopic ureterocoele, caecoureterocele, 
www.yumedtext.com | October-2018

rhabdomyosarcoma, fibroepithelial polyp, inflammatory polypoid lesions, pseudopolyps, embryonal rests, hamartomas and hemangiomas [1]. Posterior urethral polyps are rare. They have been proposed to arise from mesonephric remnants [2].

Diagnosis is usually confirmed using a combination of ultrasonography, voiding cystourethrography, and cystoscopy. Definitive management is accomplished with a variety of endoscopic techniques, including transurethral resection by cold knife [2]. There is no association with malignant degeneration.

\section{Conclusion}

Fibroepithelial polyps are a rare but important differential diagnosis of bladder masses in male infants. Rarely they present in

neonates. Presentation includes symptoms of bladder outlet obstruction, prolapsed urethral mass, voiding dysfunction or uraemia. Cystoscopic excision is the treatment of choice.

\section{REFERENCES}

1. Arena S, Barresi V, Manganaro A, et al. Bladder Mass in Newborn: Case Report and Review of Literature. Urology. 2015;86(5):1004-7.

2. Wayne T, Rapp DE, Feinstein KA, et al. Urethral polyp in asymptomatic male infant with prenatal hydronephrosis. Urology. 2006;67(5):1085.e9-11.

3. Eziyi AK, Helmy TE, Sarhan OM, et al. Management of male urethral polyps in children: Experience with four cases. Afr J Paediatr Surg. 2009;6(1):49-51.

4. Anzai Y, Koshida S, Yanagi T, et al. Neonatal urethral polyps associated with Beckwith-Wiedemann syndrome. Pediatr Int. 2013;55(5):658-61. 